\title{
BMJ Open Moving from intention to behaviour: a randomised controlled trial protocol for an app-based physical activity intervention (i2be)
}

\author{
Lili L Kókai (D) , ${ }^{1}$ Diarmaid T Ó Ceallaigh (D) , ${ }^{2,3}$ Anne I Wijtzes (D) , ${ }^{1}$ \\ Jeanine E Roeters van Lennep (D) , ${ }^{4}$ Martin S Hagger (D) , 5,6 John Cawley (D) ,2,7 \\ Kirsten I M Rohde (D) ,2,8 Hans van Kippersluis (D) ,2,3 Alex Burdorf (D) ${ }^{1}$
}

To cite: Kókai LL, 0 Ceallaigh DT, Wijtzes Al, et al. Moving from intention to behaviour: a randomised controlled trial protocol for an app-based physical activity intervention (i2be). BMJ Open 2022;12:e053711. doi:10.1136/ bmjopen-2021-053711

- Prepublication history and additional supplemental material for this paper are available online. To view these files, please visit the journal online (http://dx.doi.org/10.1136/ bmjopen-2021-053711).

LLK and DTÓC contributed equally.

Received 25 May 2021 Accepted 01 December 2021

Check for updates

(c) Author(s) (or their employer(s)) 2022. Re-use permitted under CC BY-NC. No commercial re-use. See rights and permissions. Published by BMJ.

For numbered affiliations see end of article.

Correspondence to

Lili L Kókai;

I.koka@@erasmusmc.nl

\section{ABSTRACT}

Introduction Efficacy tests of physical activity interventions indicate that many have limited or short-term efficacy, principally because they do not sufficiently build on theory-based processes that determine behaviour. The current study aims to address this limitation.

Methods and analysis The efficacy of the 8-week intervention will be tested using a three-condition randomised controlled trial delivered through an app, in women with a prior hypertensive pregnancy disorder. The intervention is based on the integrated behaviour change model, which outlines the motivational, volitional and automatic processes that lead to physical activity. The mechanisms by which the behaviour change techniques lead to physical activity will be tested.

Following stratification on baseline factors, participants will be randomly allocated in-app to one of three conditions (1:1:1). The information condition will receive information, replicating usual care. Additionally to what the information condition receives, the motivation condition will receive content targeting motivational processes. Additionally to what the motivation condition receives, the action condition will receive content targeting volitional and automatic processes.

The primary outcome is weekly minutes of moderateto-vigorous physical activity, as measured by an activity tracker (Fitbit Inspire 2). Secondary outcomes include weekly average of Fitbit-measured daily resting heart rate, and self-reported body mass index, waist-hip ratio, cardiorespiratory fitness and subjective well-being. Tertiary outcomes include self-reported variables representing motivational, volitional, and automatic processes. Outcome measures will be assessed at baseline, immediately postintervention, and at 3 and 12 months post-intervention. Physical activity will also be investigated at intervention midpoint. Efficacy will be determined by available case analysis. A process evaluation will be performed based on programme fidelity and acceptability measures.

Ethics and dissemination The Medical Ethics Committee of the Erasmus MC has approved this study (MEC2020-0981). Results will be published in peer reviewed scientific journals and presented at scientific conferences. Trial registration number Netherlands trial register, NL9329.

\section{Strengths and limitations of this study}

- Randomised controlled trial testing an $\mathrm{m}$-health intervention to promote physical activity in a population with elevated cardiovascular risk.

- Intervention is based on dual-system theory, and is using evidence-based behaviour change techniques.

- Trial tests intervention mechanisms of action by examining change in variables representing motivational, volitional and automatic processes.

- Physical activity, the primary outcome, will be measured using an activity tracker worn on the wrist (Fitbit Inspire 2).

- The study will not adopt a strict factorial design, which may limit conclusions about the exact intervention mechanisms and techniques that affect physical activity change.

\section{INTRODUCTION}

International guidelines advise adults to accumulate at least 150 minutes of moderate physical activity, or 75 minutes of vigorous physical activity, or an equivalent combination of moderate-to-vigorous intensity physical activity (MVPA) per week. ${ }^{1}$ However, over $27 \%$ of people worldwide fail to meet these guidelines. ${ }^{2}$ Insufficient MVPA has detrimental physical and mental health consequences. For instance, insufficient MVPA is a prominent behavioural risk factor for the development of cardiovascular diseases (CVDs), a leading cause of poor health and mortality worldwide. ${ }^{3}$ Therefore, the development and efficacy testing of behaviour change interventions promoting increased MVPA is highly warranted. ${ }^{4}$ If found to be efficacious, such interventions may have important implications for the design of future effectiveness trials and subsequent policy.

Behaviour change interventions promoting MVPA have been widely applied in the 
general population, and although these interventions have demonstrable efficacy, they rarely achieve large and long-term effects. ${ }^{5-7}$ Reasons for these limitations may be their lack of theoretical basis, and limited application of evidence-based behaviour change techniques. ${ }^{8}$ There is growing evidence that basing behavioural interventions on theory leads to increased efficacy in health contexts, including MVPA, ${ }^{9-11}$ and, importantly, increased precision and less variability in behaviour change relative to interventions that are not based on theory. ${ }^{12}$

Many MVPA promoting interventions are based on a single theory, typically a prominent social cognition theory (eg, protection motivation theory, theory of planned behaviour) which describes behaviour as the result of deliberative psychological processes. ${ }^{13}$ However, interventions based on such theories have generally been shown to be more effective in changing behavioural intentions than actual behaviour. ${ }^{15}$ A potential explanation for this shortcoming is that a substantive proportion of individuals hold stated intentions to perform a behaviour of interest, like MVPA, but for various reasons fail to act on them. ${ }^{16}{ }^{17}$ For example, they may forget to enact their intentions, or counter-intentional opportunities may come to light and compete with their existing intentions. ${ }^{18}$ Researchers have therefore sought to identify potential ways to promote better enactment of intentions in behavioural interventions, and minimise this 'intention-behaviour gap'. ${ }^{19}$ A further limitation of interventions based on social cognition theories is that they overlook spontaneous or impulsive behaviour that is the result of automatic processes, not directly under the conscious control or awareness of the individual. ${ }^{20} 21$

It is increasingly recognised, therefore, that researchers should base behavioural interventions on theoretical approaches that account for multiple processes that lead to action. Dual-system theories account for two types of processes that govern action: automatic processes, by which behaviour is determined by impulses and wellleaned associations between context and action, and deliberative processes, by which action is determined by reasoned deliberation and the value attached to courses of action. ${ }^{21-24}$ Integrated theories that draw their hypotheses from more than one theory have been proposed, with a view to account for these multiple processes, and to provide more comprehensive explanations of behaviour. A recent integrated theory-based approach in this vein is the integrated behaviour change (IBC) model. ${ }^{25}$ The IBC model integrates insights of multiple well-established behavioural theories to identify the multiple processes that may be implicated in MVPA. ${ }^{25-27}$

The model identifies three processes: motivational, volitional and automatic processes. The motivational processes are modelled by variables that represent deliberative decision making derived from social cognition and motivational theories. Specifically, the model specifies beliefbased variables, such as intention and attitudes from the theory of planned behaviour, and motivational variables, such as intrinsic motivation from the self-determination theory, as key behavioural determinants. In addition, the IBC model differentiates between pre-intentional (motivational) and post-intentional (volitional) processes, and, consistent with dual-phase theories, proposes that intention enactment is facilitated in the volitional phase by a planning process. Finally, the IBC model proposes that automatic processes impact behaviour beyond an individual's awareness, bypassing the intention-mediated processes. Automatic processes are represented by variables that reflect implicit decision-making, such as affect and habit. Since the development of the first IBC model, several observational studies have used it to successfully explain a number of health behaviours ranging from fat and sugar intake to sunscreen use. ${ }^{28-36}$ While these observational results are encouraging, application of the IBC model as a basis for behavioural interventions is still in its infancy and warrants further investigation.

\section{The present study}

The aim of the current study is to test the efficacy of a behavioural intervention based on the IBC model to promote MVPA. Our study population consists of women with a prior hypertensive pregnancy disorder, for whom an increase in MVPA would be particularly helpful in reducing their later-life risk for CVDs (see the Study population section). The content of the intervention will comprise health behaviour change techniques based on the IBC model, one of the first interventions to do so. ${ }^{37}$ We will systematically select behaviour change techniques closely linked to the variables described in the model (figure 1). The selection of techniques is guided by taxonomies of behaviour change techniques, ${ }^{838}$ evidence syntheses examining the association between these techniques and theoretical variables, ${ }^{39-42}$ and research showing the efficacy of these techniques in changing health behaviour. ${ }^{43-51}$

The intervention will be delivered via a smartphone application, the i2be app, using persuasive technology elements. This m-health approach was selected given evidence that smartphone-based interventions have multiple advantages over face-to-face interventions ${ }^{52}$ : they are comparatively low in cost, have a wide reach, provide flexibility in intervention location and time, and are scalable. ${ }^{53-55}$ In addition, previous online interventions using persuasive technology elements to change behaviour have demonstrated superior effects in promoting MVPA ${ }^{54} 56$ and user engagement ${ }^{5758}$ when compared with interventions that did not use such elements.

The study will have three conditions. Each condition will include different sets of behaviour change techniques aimed at tapping into the different processes identified in the IBC model. This study design will provide insight into the relative efficacy of groups of techniques that target change in the variables that represent the motivational, volitional, and automatic processes that predict behaviour based on the IBC model.

The study population will consist of women with a prior hypertensive pregnancy disorder. It is hypothesised 


\section{Behavior Change Techniques Processes Mechanism of Action Variables}

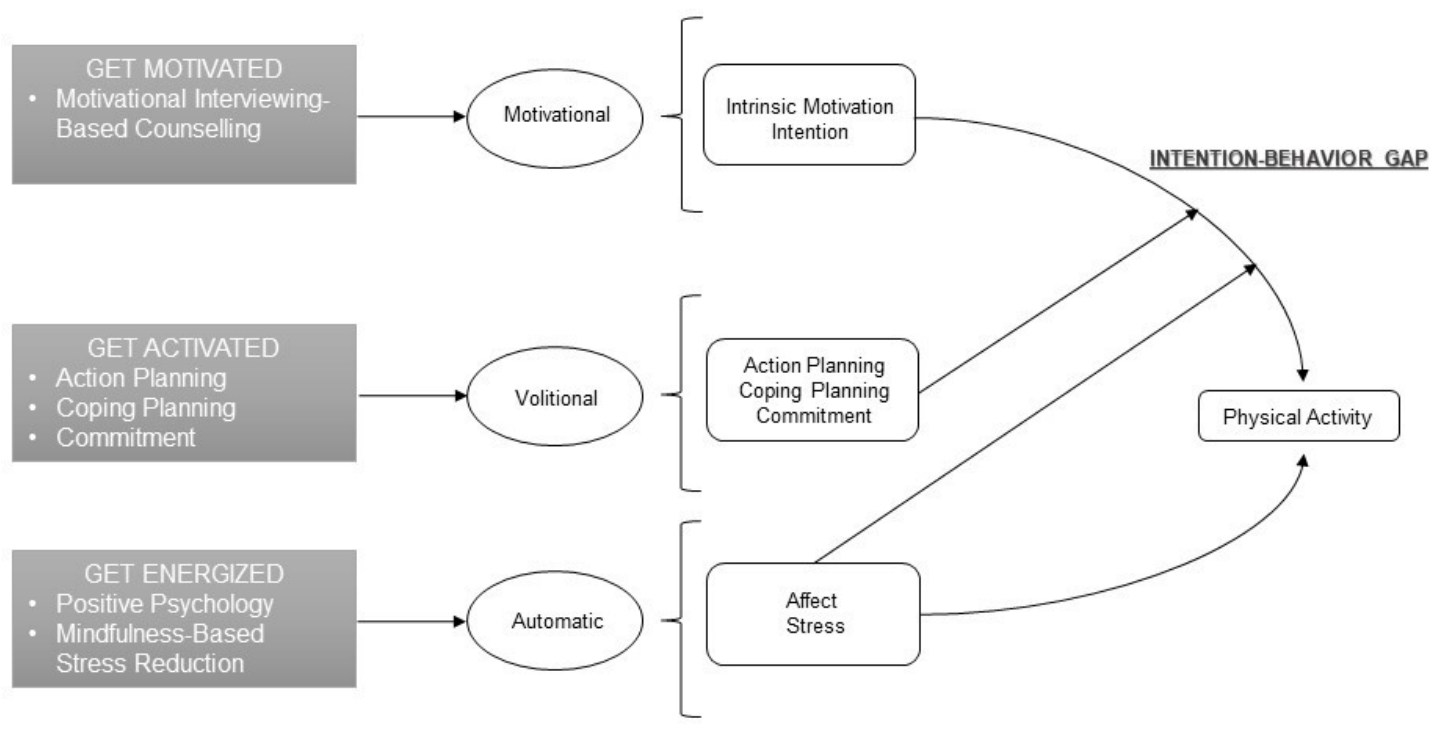

Figure 1 The integrated behaviour change model and intention-behaviour gap.

that women who develop such complications have unrevealed CVD risk factors prior to pregnancy, which are then exacerbated by the metabolic stress of pregnancy, consequently contributing to the occurrence of hypertensive pregnancy disorders. ${ }^{59}$ Hypertensive pregnancy disorders, such as pre-eclampsia, eclampsia and haemolysis, elevated liver enzymes, low platelet count (HELLP) syndrome, are cardiometabolic risk factors for CVDs. ${ }^{60} 61$ For example, women with pre-eclampsia have a twofold to sevenfold increased risk for CVDs later in life relative to women with a normotensive pregnancy. ${ }^{62} 63$

We have decided to use this study population, that is, women with prior hypertensive pregnancy disorders, for several reasons. First, an MVPA intervention may provide an especially high social return in this population due to these women's increased risk for CVDs later in life. ${ }^{6164-66}$ Second, these women are likely to be motivated to participate in an MVPA intervention, partly due to the "window of opportunity' that their new motherhood presents, and partly due to their elevated risk for CVDs later in life. Third, these women typically do not have any physical limitations that would prevent their participation in an MVPA intervention, as other patient groups with a high CVD risk might have. Finally, these women are relatively young and healthy (their increased risk for CVDs is not likely to show until later in life), which may make our findings generalisable to other young and healthy adult populations.

\section{METHODS AND ANALYSIS}

The protocol follows the 'Standard Protocol Items: Recommendations for Interventional Trials' guidelines. ${ }^{67}$

\section{Study population}

Given that the associated CVD risk among women with a prior hypertensive pregnancy disorder is largest for those with a history of pre-eclampsia, especially severe pre-eclampsia, we aim to first draw participants from this population. ${ }^{62}{ }^{63}$ In the Netherlands, unique cardiovascular follow-up and care is provided to women with prior severe pre-eclampsia at the multidisciplinary Follow-Up Pre-Eclampsia Outpatient Clinic (FUPEC) of the Erasmus MC, the only clinic of its kind in the country. ${ }^{68}$ There are currently around 1000 patients registered in the clinic, with an additional 100 to 150 women enrolling each year. In case the intended sample size $(\mathrm{N}=630$; see the Sample size calculation section) cannot be fully recruited from the FUPEC clinic, further recruitment will take place, first through the official Dutch patient organisation for women with pre-eclampsia and/or HELLP syndrome (HELLP foundation). Second, we would then recruit women with other prior hypertensive pregnancy disorders through the Department of Gynaecology and Obstetrics of the Erasmus MC, and other hospitals in the Netherlands.

Inclusion criteria for enrollment is having been diagnosed with a hypertensive pregnancy disorder in the past. Exclusion criteria for enrollment into the trial are: $<18$ years of age, pregnant at time of inclusion, $<3$ months after delivery, any physical health limitations preventing MVPA (eg, illness, injury, surgery, rehabilitation), no working knowledge of Dutch or English language, and no possession of a smartphone. Invited women are informed that participation in the trial is voluntary. Women who choose to participate will be asked to sign an informed consent form in advance of participation. Participants will be informed that they may leave the study at any point in time without having to provide a reason. Following drop-out, no further data will be collected. Participants dropping out of the study will not be substituted. 


\section{Patient and public involvement}

Patients of the FUPEC clinic have been involved in the design of the i2be app. Through a qualitative survey $(\mathrm{N}=35)$, we have gained qualitative information on the processes described by the IBC model, and on the needs of the population in terms of m-health intervention delivery. Patients and members of the public will not be involved in the conduct or reporting of this study.

\section{Design}

The intervention will last for 8 weeks, and outcome measures will be collected at four time points: baseline, immediately post-intervention, and at 3 and 12 months post-intervention. MVPA will additionally be investigated at the intervention midpoint, that is, 4 weeks after the start of the intervention. The study will adopt a threecondition randomised controlled design. Participants will be stratified on time since pregnancy $(<12$ months postpartum vs $\geq 12$ months postpartum), and self-reported average weekly minutes of MVPA in the last month (low: $\mathrm{x}<2.5$ hours; medium: 2.5 hours $\leq \mathrm{x}<7$ hours; high: 7 hours $\leq \mathrm{x})$, and randomly allocated (1:1:1) in-app to one of the three parallel intervention conditions (the information condition, the motivation condition, or the action condition) using permuted block randomisation (using variable block sizes of six or nine). Participants will not be directly informed of the condition to which they have been allocated, but as participants will be presented with the content of the intervention, they cannot be considered blind to allocation. Randomisation to intervention condition is carried out automatically by the app, and the data on allocation is held securely by the app developers, Avegen (a digital health company). As this data is not accessible by the research team during the 8-week intervention period and while outcome measures are being collected immediately post-intervention, the research team will be blind to the allocation of participants during that period.

This study design will allow us to gain insight into the incremental effect of behaviour change techniques that target variables representing the volitional and automatic processes in the action condition, in addition to the effect of techniques that target variables representing the motivational processes in the motivation condition. The size of this incremental effect will be benchmarked against the incremental effect of targeting motivational processes alone in the motivation condition, and the provision of information only in the information condition. The information condition will replicate usual care, as it contains information that women with a prior hypertensive pregnancy disorder receive from their healthcare provider.

\section{Intervention conditions}

Participants in the information condition receive the 'Get Informed' module, which provides them with information on topics related to MVPA. First, the relationship between hypertensive pregnancy disorders, increased risk of CVDs, and MVPA is explained. Second, WHO guidelines for
MVPA are presented, examples of MVPA are given, and it is explained how Fitbit devices measure MVPA. Finally, recommendations on how to warm-up, cool-down, lower risk of injury, and achieve all-round health and well-being are provided. This content largely corresponds to the usual care offered to women with prior hypertensive pregnancy disorders, and primarily serves to stimulate those in the information condition to use the app.

In addition to the 'Get Informed' module, participants in the motivation condition will receive the 'Get Motivated' module, which targets motivational processes, and consists of motivational interviewing-based counselling techniques. Participants will receive an interactive, fully automated activity each week, consisting of content-based motivational interviewing techniques. ${ }^{69}$

In addition to receiving the 'Get Informed' and 'Get Motivated' modules, participants in the action condition will receive the 'Get Activated' module, which targets volitional processes, consisting of action planning, coping planning, and commitment techniques. Participants will receive interactive, fully automated activities each week, aiding them in setting a self-defined weekly MVPA goal, committing to that goal with i2be points, making action plans and coping plans to reach that goal, and making their own self-defined commitments outside of i2be. ${ }^{470}$ Furthermore, participants in the action condition will receive the 'Get Energised' module, which targets automatic processes, and comprises mindfulness-based stress reduction and positive psychology techniques. Participants will receive 4 minute audio clips of mindfulnessbased stress reduction, and interactive, fully automated positive psychology exercises (table 1). ${ }^{48} 495171$

\section{Outcome measures}

The primary outcome of this research project is weekly minutes of MVPA, as measured by an activity tracker worn on the wrist (Fitbit Inspire 2). Fitbit devices are well suited for the measurement of MVPA (and resting heart rate) in the context of MVPA interventions due to their relative accuracy. ${ }^{72}$ Naturally, Fitbit-measured data is likely to be imperfect due to possibilities of missing or incomplete data arising from intentional (eg, aversion to Fitbit device use) or unintentional non-compliance (eg, forgetting to wear Fitbit device), and measurement error in data (eg, imperfect measurement of MVPA).

Secondary outcomes consist of Fitbit-measured weekly average of daily resting heart rate, as well as self-reported body mass index, waist-hip ratio, cardiorespiratory fitness, and subjective well-being. The outcome measures of body mass index and waist-hip ratio will be combined into one overweight index by standardising both measures and getting the average of these two standardised measures. Tertiary outcomes include self-reported motivation, intention, action planning, coping planning, commitment, affect and stress. Control variables measured include selfreported trait self-control, habit, age, education, household composition, type of prior hypertensive pregnancy 


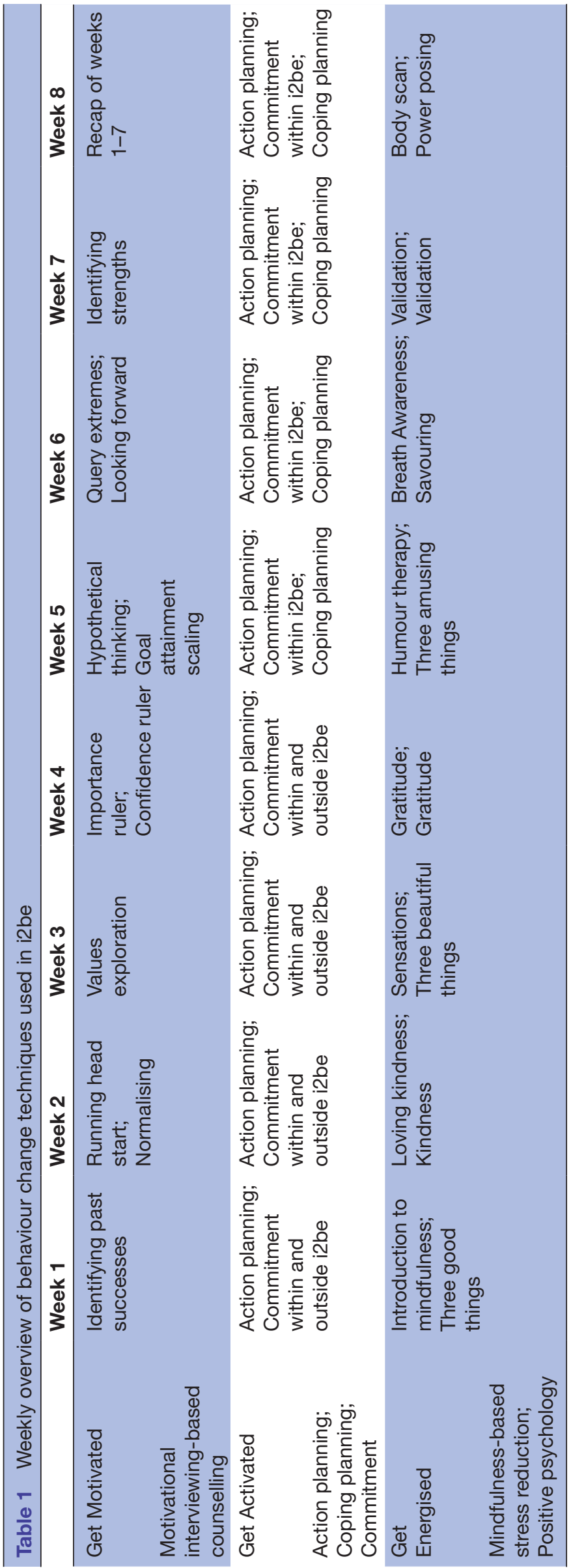


disorder and whether the participant is currently pregnant or lactating (table 2).

\section{Implementation}

A rolling recruitment and enrollment procedure will be used, initially aiming for a 6-week time frame (September to November 2021), which may be extended depending on when the target sample size is met. The first participants are expected to complete the 8-week intervention in early December 2021. An invitation email containing a 2 minute long introduction video and the participant information letter of the i2be study will be sent out to potential participants by healthcare professionals, in cases where recruitment is through the hospital, or the i2be research team, in cases where recruitment is through the patient organisation. Patients interested in participating are directed to a short online questionnaire to assess eligibility based on study inclusion and exclusion criteria. Eligible patients are then asked to provide informed consent for participation, as well as their contact information. They will then receive an email containing the following information: that their Fitbit Inspire 2 will be sent to them by mail, a link to download the i2be app and the Fitbit app from the Google Play Store (for Android smartphones) or App Store (for iPhones), and a Welcome Pack. The Welcome Pack will detail the technical setup necessary for participation (ie, pairing the Fitbit app to the Fitbit device and the i2be app, syncing the Fitbit app weekly, using the Fitbit device, general i2be app structure).

When using the i2be app for the first time, participants will be asked to choose their preferred language (Dutch or English) and register. Subsequently, participants will be stratified and randomly allocated in-app to one of the three intervention conditions, as previously stated. Participants know that there are three versions of the app and that they are in one of them, but they do not know which one (ie, information, motivation or action). An up to 2-week window is provided for technical setup before the baseline measurement. During this pre-baseline measurement period, participants have the option to watch the i2be introduction video, to have a wizard take them through the main functionalities of the app (left column under Onboarding in figure 2), and have access to the general tabs of the app (Other Tabs in figure 2). From the start of the baseline measurement, participants can log in to the app directly (right column under Onboarding in figure 2), and from then onwards can access all of their allocation features (including the Home Tab in figure 2). The 8-week intervention period will start once the participant has completed their baseline measurement, that is, completed the self-reported outcome measures, worn their Fitbit device for the baseline measurement week, and synchronised their Fitbit app at the end of that week. During the intervention period participants are expected to spend 15-30 minutes per week on module content. Furthermore, they are expected to spend an additional 15-30 minutes on completing outcome measurements at four time points, that is, baseline, immediately postintervention, and at 3 and 12 months post-intervention.

\section{User interface}

For all participants, the user interface of the i2be app shows a diverse range of women of different ethnic backgrounds and ages representative of the population, and uses female voice-overs in the introduction video and audio clips. Participants can find basic app functionalities, such as terms and conditions, under the Menu tab. The 'Get Informed', 'Get Motivated', 'Get Activated' and 'Get Energised' modules consist of interactive, fully automated activities each week, which will appear on the Home tab. Outcome measures to be filled in will also appear on the Home tab. Participants will be reminded weekly to sync the Fitbit app, also on the Home tab. The Notifications tab will announce the release of weekly activities, and reminders of weekly activities. Participants will be able to view their recent results of Fitbit-measured weekly minutes of MVPA and daily resting heart rate, and self-reported body mass index and waist-hip ratio under the My Health tab. They will also be able to track their progress related to i2be points under the My Progress tab (see Persuasive technology elements and Gamification below).

\section{Persuasive technology elements}

Two types of persuasive technology elements will be used by the i2be app: primary task support and dialogue support. ${ }^{57}$ Not all elements will be present in all conditions, consistent with the study design. Primary task support involves reduction, personalisation and selfmonitoring elements. ${ }^{57}$ The reduction and personalisation elements involve the self-setting of weekly MVPA goals. Some further personalised elements of the app include recaps of participants' past responses, the choice of when to receive a reminder of self-planned MVPA, and the option to commit to MVPA goals. The My Health and My Progress tabs both allow for self-monitoring. Dialogue support is provided to participants through reminders, suggestions, praise and rewards. ${ }^{57}$ More specifically, participants receive a reminder of their action plan(s). Participants receive a reminder of their action plan(s) 1 hour prior to the planned MVPA by default (which they can adapt to a timing of their own liking). Participants receive automated praise for completing activities. Finally, participants will receive various rewards for participation (see the Gamification section).

\section{Gamification}

For all participants, the completion of activities is linked to a virtual point system (i2be points), resulting in psychological rewards and tangible rewards. After every 100 i2be points accumulated, participants receive the psychological reward of progressing to the next level of achievement in-app (Bee Levels). The virtual i2be points are further used to qualify for tangible rewards. Participants enter into a weekly raffle for a self-selected sports 
Table 2 Schematic overview of data collection during the trial

\begin{tabular}{|c|c|c|c|c|}
\hline Outcomes & Variables & Measurements & Baseline & Follow-up* \\
\hline \multicolumn{5}{|l|}{ Primary outcome } \\
\hline $\begin{array}{l}\text { Objectively measured by } \\
\text { Fitbit }\end{array}$ & Physical activity $†$ & $\begin{array}{l}\text { Moderate-to-vigorous intensity physical } \\
\text { activity (MVPA) (min/week) }\end{array}$ & $\checkmark$ & $\checkmark$ \\
\hline \multicolumn{5}{|l|}{ Secondary outcomes } \\
\hline $\begin{array}{l}\text { Objectively measured by } \\
\text { Fitbit }\end{array}$ & Heart rate $†$ & $\begin{array}{l}\text { Week average of daily resting heart rate } \\
\text { (beats/min) }\end{array}$ & $\checkmark$ & $\checkmark$ \\
\hline \multirow[t]{4}{*}{ Self-reported into app } & Body mass index $\ddagger$ & Weight/length ${ }^{2}\left(\mathrm{~kg} / \mathrm{m}^{2}\right)$ & $\checkmark$ & $\checkmark$ \\
\hline & Waist-hip ratio $\neq$ & Waist circumference/hip circumference & $\checkmark$ & $\checkmark$ \\
\hline & Cardiorespiratory fitness & 1 mile Rockport walk test & $\checkmark$ & $\checkmark$ \\
\hline & Subjective well-being & Satisfaction with Life Scale & $\checkmark$ & $\checkmark$ \\
\hline \multicolumn{5}{|l|}{ Tertiary outcomes } \\
\hline \multirow[t]{7}{*}{ Self-reported into app } & Motivation & $\begin{array}{l}\text { The Behavioural Regulation in Exercise } \\
\text { Questionnaire }\end{array}$ & $\checkmark$ & $\checkmark$ \\
\hline & Intention & Own design based on Ajzen guidelines & $\checkmark$ & $\checkmark$ \\
\hline & Action planning & Own design based on Sniehotta measure & $\checkmark$ & $\checkmark$ \\
\hline & Coping planning & Own design based on Sniehotta measure & $\checkmark$ & $\checkmark$ \\
\hline & Commitment & Own design & $\checkmark$ & $\checkmark$ \\
\hline & Affect & Global Mood Scale & $\checkmark$ & $\checkmark$ \\
\hline & Stress & Perceived Stress Scale & $\checkmark$ & $\checkmark$ \\
\hline \multicolumn{5}{|l|}{ Control variables } \\
\hline \multirow[t]{8}{*}{ Self-reported into app } & Trait self-control & Brief Self-control Scale & $\checkmark$ & \\
\hline & Habit & Habit strength & $\checkmark$ & \\
\hline & Age & Age (years) & $\checkmark$ & \\
\hline & Education & $\begin{array}{l}\text { English version based on ISCED } 2011 \\
\text { Dutch version based on SOI } 2016\end{array}$ & $\checkmark$ & \\
\hline & Household composition & Living situation (partner, children) & $\checkmark$ & \\
\hline & Lactation status & Currently lactating (yes/no) & $\checkmark$ & \\
\hline & Pregnancy status & Currently pregnant (yes/no; due date) & $\checkmark$ & $\checkmark$ \\
\hline & Type of disorder & Type of hypertensive pregnancy disorder & $\checkmark$ & \\
\hline
\end{tabular}

\begin{tabular}{|c|c|c|c|c|c|}
\hline \multicolumn{6}{|l|}{ Preferences } \\
\hline Self-reported into app & Voucher preference & Choice from three sports store vouchers & $\checkmark$ & & \\
\hline \multicolumn{6}{|l|}{ Stratification variables } \\
\hline Self-reported into app & MVPA & $\begin{array}{l}\text { Average weekly minutes of MVPA in the } \\
\text { past month (low } / \mathrm{mid} / \mathrm{high} \text { ) }\end{array}$ & $\checkmark$ & & \\
\hline Self-reported into app & Programme acceptability & $\begin{array}{l}\text { Component usability, appropriateness, } \\
\text { engagement, appeal, satisfactions and } \\
\text { dissatisfactions }\end{array}$ & & $\checkmark$ & $\S$ \\
\hline $\begin{array}{l}\text { Objectively measured by } \\
\text { app }\end{array}$ & Programme fidelity & Compliance with programme & $\checkmark$ & $\checkmark$ & \\
\hline
\end{tabular}

*Follow-up measurements immediately post-intervention, and at 3 and 12 months post-intervention.

†Also measured weekly for the duration of the 8-week intervention.

$\ddagger$ Body mass index and waist-hip ratio are combined into one overweight index by standardising both measures and taking their average.

§Only measured immediately post-intervention.

ISCED, International Standard Classification of Education; SOI, Standaard Onderwijsindeling. 


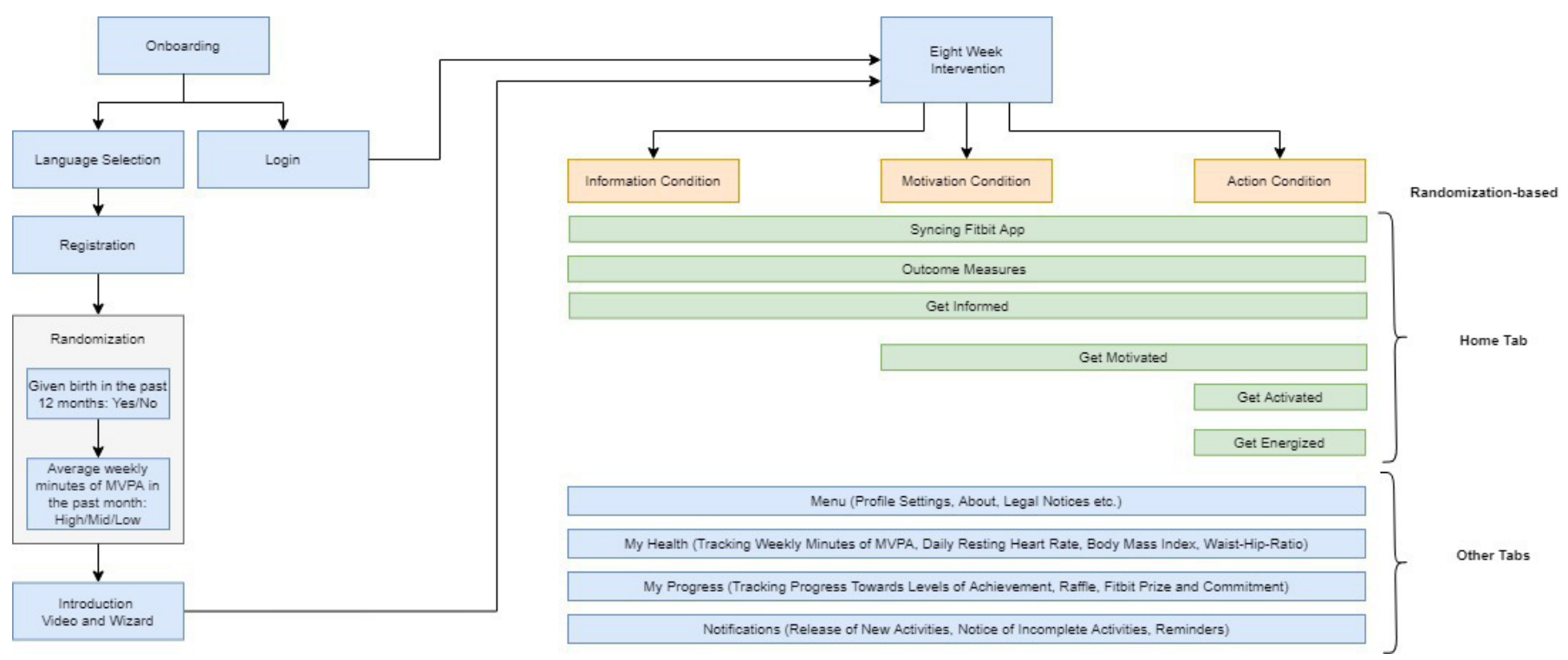

Figure 2 Allocation flow chart. MVPA: moderate-to-vigorous intensity physical activity.

store voucher (worth €25-€30) under the condition that they earn the maximum i2be points for that week. Lastly, conditional on reaching a certain threshold of accumulated i2be points, participants can keep their Fitbit device at the end of the intervention.

In addition to the psychological and tangible rewards available to all participants, participants in the action condition can choose to commit to their MVPA goal of that week, that is, deposit some of their i2be points. If they achieve their MVPA goal for that week, their i2be points are returned to them, and they will also be eligible to take part in that week's raffle, conditional on their compliance with all other activities. However, if they do not achieve their MVPA goal for that week, they lose their deposited i2be points, and their eligibility for that week's raffle (figures 2 and 3 ).

\section{Sample size calculation}

A meta-analysis of recent smartphone-based physical activity interventions found a small-to-medium average effect size $(\mathrm{d}=0.31)$ on daily minutes of physical activity. ${ }^{73}$ We carried out our sample size calculation for this study under the assumption that this average effect size is achieved both in testing the action condition against the information condition, and in testing the motivation condition against the information condition. Given this, we aim to recruit 630 participants to the trial with uniform randomisation across conditions (ie, 210 participants per condition). The average attrition rate in previous webbased physical activity interventions was 20\%. ${ }^{74}$ Such an attrition rate would leave us with a final total sample size of 504. Taking into account the potential maximum deviation from a 1:1:1 allocation ratio which may arise due to the stratified block randomisation procedure, our intended sample size $(\mathrm{N}=630)$ will give us sufficient statistical power (alpha $=0.05$, power $=0.8$ ) at our primary time point (immediately post-intervention) to detect effect sizes greater than or equal to the average effect size found in the meta-analysis $(d=0.31)$. The study will also be adequately powered to detect such effect sizes at the 3 and 12 months post-intervention time points provided that overall attrition does not exceed $20 \%$ at those time points. This should be adequate to detect the effect size when testing each of the action and motivation conditions against the information condition. The effect size when testing the action condition against the motivation condition may be smaller than $\mathrm{d}=0.31$, and thus we may be underpowered to detect this effect.

\section{Data analysis plan}

The primary analysis will be an available case analysis (ie, participants with missing dependent variable data are excluded). Our primary outcome at each time point will be total minutes of MVPA for the full measurement week at that time point (eg, for the immediately postintervention time point, this is the 7 days immediately post-intervention) as measured by the Fitbit activity tracker.

Linear regression will be used to assess differences between groups at each time point. The first test of i2be is the difference between the action condition and the information condition to see whether our intervention as a whole is of value for the promotion of MVPA. Second, we will test the difference between the action condition and the motivation condition to gain insight into the added value of targeting volitional and automatic processes above targeting motivational processes to promote MVPA. Third, we will test the difference between the motivation condition and the information condition to produce an effect size value against which the main test of the efficacy of the i2be intervention can be benchmarked. Sensitivity analyses will be carried out to assess the robustness of results to the missing data strategy adopted by carrying out intention-to-treat analyses using imputation methods (multiple imputation by chained equations, ${ }^{75}$ best-worst and worst-best analysis ${ }^{76}$ ), 

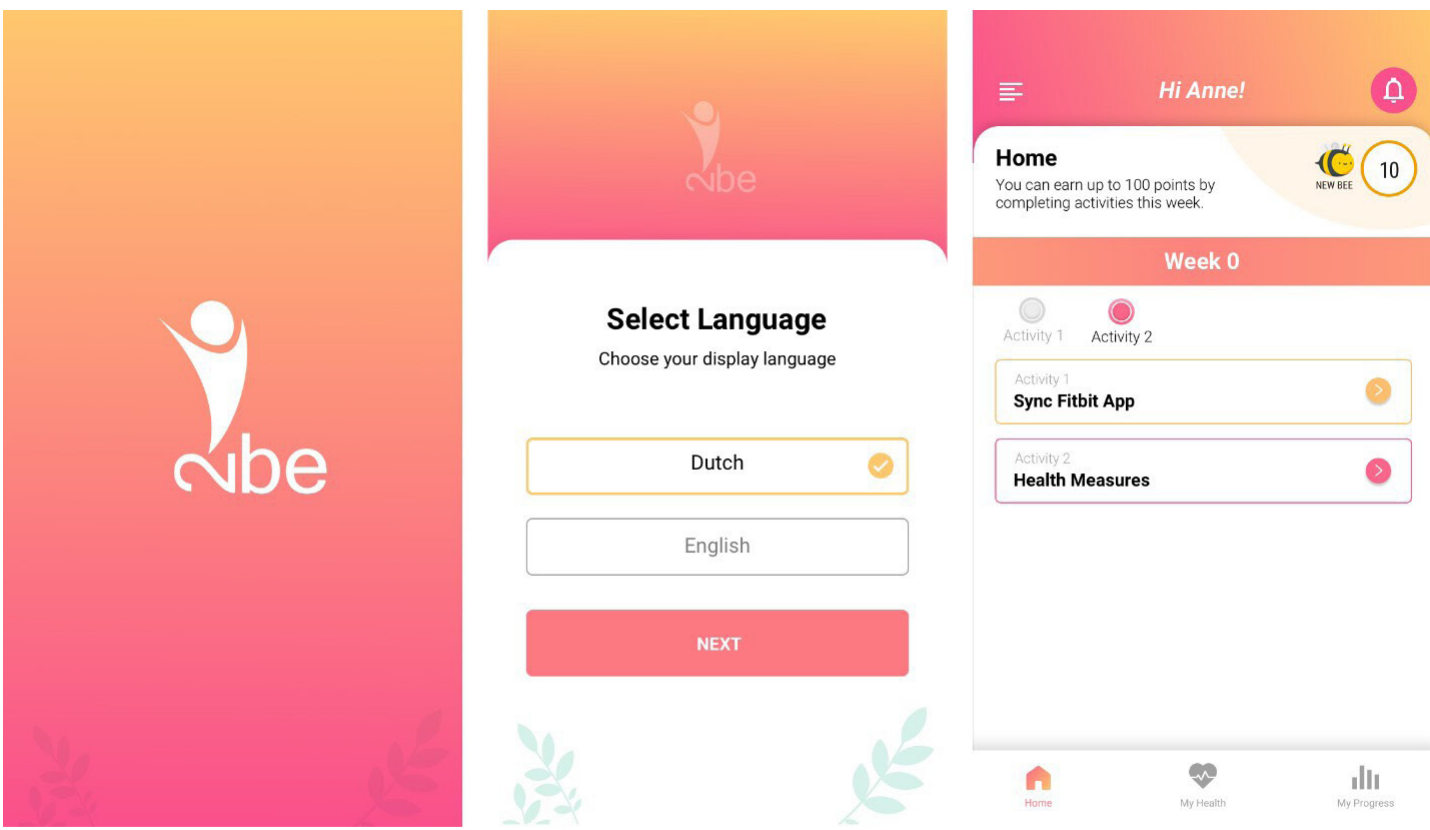

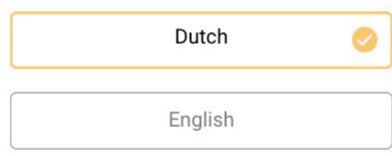

Select Language

Choose your display language
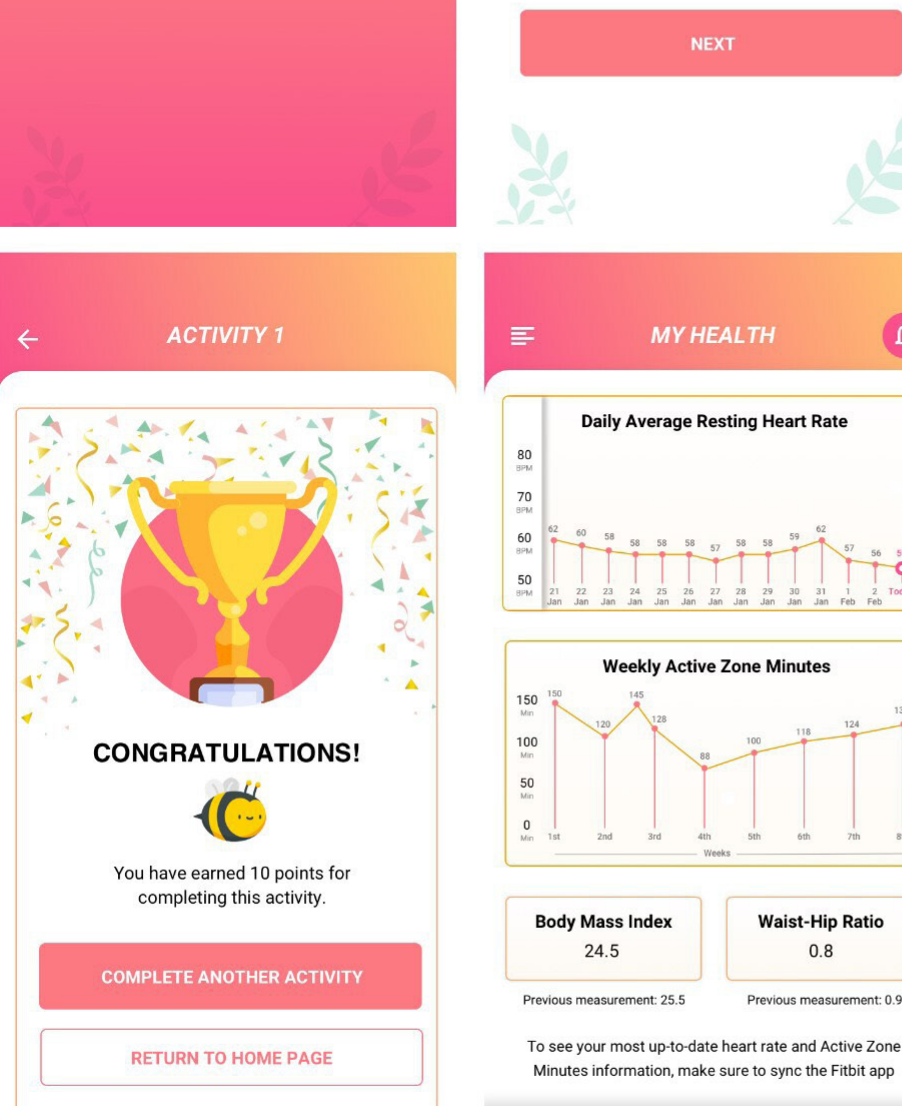
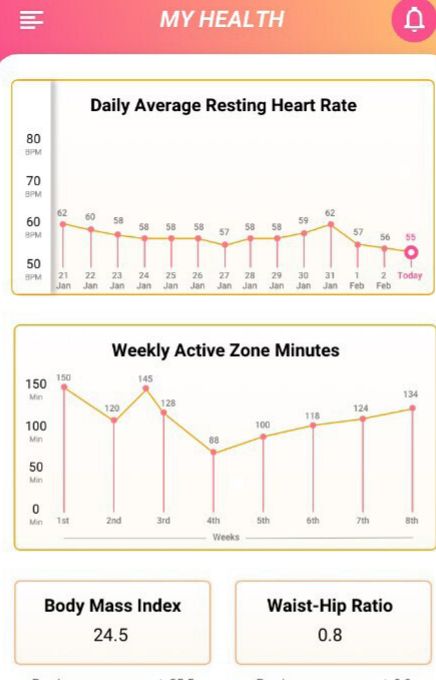
0.8

Previous measurement 25.5

Previous measurement 0.9

To see your most up-to-date heart rate and Active Zone Minutes information, make sure to sync the Fitbit app

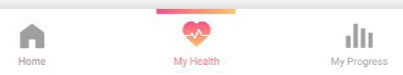

Waist-Hip Ratio

Figure 3 Look and feel of the i2be environment.

and when attrition is high and non-random by carrying out per-protocol analysis. To assess whether our intervention has differential effects across educational levels, subgroup analysis by educational level will be performed. Data analyses will be adjusted for control variables to decrease potential residual confounding after randomisation, and to increase statistical power (online supplemental file).

A process evaluation of the intervention will be performed immediately after the intervention, using programme fidelity measures (ie, user engagement statistics collected by the app such as how many people complete all module content and each individual module, and how the module completion rates change over time), and programme acceptability measures (ie, assessing self-reported component usability, appropriateness, engagement and appeal, and other satisfactions and dissatisfactions).

\section{Data management}

Data will be handled confidentially and stored in a pseudonymised manner. The identification key linking unique participant ID with personal data will be safeguarded and kept separate from deidentified research data. The 
identification key, informed consent forms, and deidentified research data will be archived for at least 10 years after completion of the study.

\section{Ethics and dissemination}

The study will adhere most strictly to all applicable legal, ethical, and safety provisions of the Netherlands and the EU. The study will be conducted in accordance with the principles of the Declaration of Helsinki. ${ }^{77}$ The Medical Ethics Committee of the Erasmus MC has approved this study (MEC-2020-0981). Findings from the study will be presented at national and international scientific conferences. Furthermore, articles reporting on these findings will be submitted for publication in leading international peer-reviewed scientific journals. Results will be communicated to the general public through general conferences, meetings and newsletters.

\section{Individual participant data sharing plan}

On completion of the trial, and after the publication of the results, researchers who provide a methodologically sound proposal can request individual deidentified participant-level data from the corresponding author for those participants who have provided informed consent for sharing of data.

\section{Author affiliations}

${ }^{1}$ Department of Public Health, Erasmus MC University Medical Center, Rotterdam, The Netherlands

${ }^{2}$ Erasmus School of Economics, Erasmus University Rotterdam, Rotterdam, The Netherlands

${ }^{3}$ Tinbergen Institute, Erasmus University Rotterdam, Rotterdam, The Netherlands ${ }^{4}$ Department of Internal Medicine, Erasmus MC University Medical Center, Rotterdam, The Netherlands

${ }^{5}$ Department of Psychological Sciences, University of California Merced, Merced, California, USA

${ }^{6}$ Faculty of Sport and Health Sciences, University of Jyväskylä, Jyväskylä, Finland ${ }^{7}$ Department of Policy Analysis and Management, Cornell University, Ithaca, New York, USA

${ }^{8}$ Tinbergen Institute and Erasmus Research Institute of Management, Erasmus University Rotterdam, Rotterdam, The Netherlands

Twitter Lili L Kókai @lili_kokai, Diarmaid T ó Ceallaigh @Diarmaid0Ceall1, Jeanine E Roeters van Lennep @j_rvl and John Cawley @cawley_john

Acknowledgements We would like to thank Aurelien Baillon, Arnold Bakker, Michele Belot, Ad Bergsma, Marte van der Bijl, Han Bleichrodt, Johannes Duvekot, Daphne Jansen, Vincent Kortleve, Geert Lonterman, Johan Mackenbach, Owen O'Donnell, Heather Royer, Getoar Sopa, Ruut Veenhoven, and Elisa de Weerd for their contribution to the content development of the app. The i2be app has been developed in collaboration with Avegen, a digital health company that aims to empower individuals to take control of their health and has specific expertise in the areas of cardiovascular health, maternal health, and individualised care.

Contributors LLK: conceptualisation, methodology, writing first draft of the protocol, revising the protocol; DTOC: conceptualisation, methodology, preanalysis plan, reviewing drafts of the protocol; AIW: conceptualisation, methodology, supervision, reviewing drafts of the protocol; JERvL: methodology, clinical guidance, reviewing drafts of the protocol; MSH: conceptualisation, methodology, reviewing drafts of the protocol; JC: conceptualisation, methodology, reviewing drafts of the protocol; KIMR: conceptualisation, methodology, supervision, reviewing drafts of the protocol; HvK: conceptualisation, methodology, supervision, reviewing drafts of the protocol; $A B$ : conceptualisation, methodology, supervision, reviewing drafts of the protocol; all authors read and approved the final version of the protocol.
Funding This study was funded by the Smarter Choices for Better Health Initiative (Action Line Prevention), grant number 108348, and the Erasmus Trustfonds, grant number 97030.2020.101.260/199/MB.

Competing interests None declared.

Patient consent for publication Not applicable.

Provenance and peer review Not commissioned; externally peer reviewed.

Supplemental material This content has been supplied by the author(s). It has not been vetted by BMJ Publishing Group Limited (BMJ) and may not have been peer-reviewed. Any opinions or recommendations discussed are solely those of the author(s) and are not endorsed by BMJ. BMJ disclaims all liability and responsibility arising from any reliance placed on the content. Where the content includes any translated material, BMJ does not warrant the accuracy and reliability of the translations (including but not limited to local regulations, clinical guidelines, terminology, drug names and drug dosages), and is not responsible for any error and/or omissions arising from translation and adaptation or otherwise.

Open access This is an open access article distributed in accordance with the Creative Commons Attribution Non Commercial (CC BY-NC 4.0) license, which permits others to distribute, remix, adapt, build upon this work non-commercially, and license their derivative works on different terms, provided the original work is properly cited, appropriate credit is given, any changes made indicated, and the use is non-commercial. See: http://creativecommons.org/licenses/by-nc/4.0/.

\section{ORCID iDs}

Lili L Kókai http://orcid.org/0000-0003-1960-3939

Diarmaid T ó Ceallaigh http://orcid.org/0000-0002-5228-6739

Anne I Wijtzes http://orcid.org/0000-0001-8335-6106

Jeanine E Roeters van Lennep http://orcid.org/0000-0001-6870-9962

Martin S Hagger http://orcid.org/0000-0002-2685-1546

John Cawley http://orcid.org/0000-0002-4805-9883

Kirsten I M Rohde http://orcid.org/0000-0002-0222-7474

Hans van Kippersluis http://orcid.org/0000-0003-3297-5059

Alex Burdorf http://orcid.org/0000-0003-3129-2862

\section{REFERENCES}

1 WHO. Global recommendations on physical activity for health: 18-64 year olds, 2011.

2 Guthold R, Stevens GA, Riley LM, et al. Worldwide trends in insufficient physical activity from 2001 to 2016: a pooled analysis of 358 population-based surveys with 1.9 million participants. Lancet Glob Health 2018;6:e1077-86.

3 Virani SS, Alonso A, Benjamin EJ, Salim SV, Alvaro A, Emelia JB, et al. Heart disease and stroke Statistics-2020 update: a report from the American heart association. Circulation 2020;141:139-596.

4 Thomas H, Diamond J, Vieco A. Global atlas of cardiovascular disease. Glob Heart 2018;13:143-63.

5 Romeo A, Edney S, Plotnikoff R, et al. Can smartphone apps increase physical activity? systematic review and meta-analysis. $J$ Med Internet Res 2019;21:e12053.

6 Murray JM, Brennan SF, French DP, et al. Effectiveness of physical activity interventions in achieving behaviour change maintenance in young and middle aged adults: a systematic review and metaanalysis. Soc Sci Med 2017;192:125-33.

7 Direito A, Carraça E, Rawstorn J, et al. mHealth technologies to influence physical activity and sedentary behaviors: behavior change techniques, systematic review and meta-analysis of randomized controlled trials. Ann Behav Med 2017;51:226-39.

8 Michie S, Carey RN, Johnston M, et al. From theory-inspired to theory-based interventions: a protocol for developing and testing a methodology for linking behaviour change techniques to theoretical mechanisms of action. Ann Behav Med 2018;52:501-12.

9 Protogerou C, Johnson BT. Factors underlying the success of behavioral HIV-prevention interventions for adolescents: a metareview. AIDS Behav 2014;18:1847-63.

10 Bishop FL, Fenge-Davies AL, Kirby S, et al. Context effects and behaviour change techniques in randomised trials: a systematic review using the example of trials to increase adherence to physical activity in musculoskeletal pain. Psychol Health 2015;30:104-21.

11 Webb TL, Joseph J, Yardley L, et al. Using the Internet to promote health behavior change: a systematic review and meta-analysis of the impact of theoretical basis, use of behavior change techniques, and mode of delivery on efficacy. J Med Internet Res 2010;12:e4. 
12 McEwan D, Beauchamp MR, Kouvousis C, et al. Examining the active ingredients of physical activity interventions underpinned by theory versus no stated theory: a meta-analysis. Health Psychol Rev 2019;13:1-17.

13 Painter JE, Borba CPC, Hynes M, et al. The use of theory in health behavior research from 2000 to 2005: a systematic review. Ann Behav Med 2008;35:358-62.

14 Webb TL, Sheeran P. Does changing behavioral intentions engender behavior change? A meta-analysis of the experimental evidence. Psychol Bull 2006;132:249-68.

15 Sniehotta FF, Scholz U, Schwarzer R. Bridging the intentionbehaviour gap: planning, self-efficacy, and action control in the adoption and maintenance of physical exercise. Psychol Health 2005;20:143-60.

16 Orbell S, Sheeran P. 'Inclined abstainers': a problem for predicting health-related behaviour. Br J Soc Psychol 1998;37:151-65.

17 Rhodes RE, de Bruijn G-J. How big is the physical activity intentionbehaviour gap? A meta-analysis using the action control framework. Br J Health Psychol 2013;18:296-309.

18 Sheeran P, Webb TL. The Intention-Behavior gap. Soc Personal Psychol Compass 2016:10:503-18.

19 Bélanger-Gravel A, Godin G, Amireault S. A meta-analytic review of the effect of implementation intentions on physical activity. Health Psychol Rev 2013;7:23-54.

20 Thinking KD. Fast and slow. New York: Farrar, Straus and Giroux, 2011.

21 Hagger MS. Non-conscious processes and dual-process theories in health psychology. Health Psychol Rev 2016;10:375-80.

22 Loewenstein G. Out of control: visceral influences on behavior. Organ Behav Hum Decis Process 1996;65:272-92.

23 de Ridder DTD, Lensvelt-Mulders G, Finkenauer C, et al. Taking stock of self-control: a meta-analysis of how trait self-control relates to a wide range of behaviors. Pers Soc Psychol Rev 2012;16:76-99.

24 Hofmann W, Friese M, Wiers RW. Impulsive versus reflective influences on health behavior: a theoretical framework and empirical review. Health Psychol Rev 2008;2:111-37.

25 Hagger MS, Chatzisarantis NLD. An integrated behavior change model for physical activity. Exerc Sport Sci Rev 2014;42:62-9.

26 Ajzen I. The theory of planned behavior. Organ Behav Hum Decis Process 1991;50:179-211.

27 Deci ELR RM. Self-determination theory: when mind mediates behavior. J Mind Behav 1980;1:33-43.

28 Hagger MS, Trost N, Keech JJ, et al. Predicting sugar consumption: application of an integrated dual-process, Dual-phase model. Appetite 2017;116:147-56.

29 Hamilton K, Kirkpatrick A, Rebar A, et al. Child sun safety: application of an integrated behavior change model. Health Psychol 2017:36:916-26.

30 Brown DJ, Hagger MS, Morrissey S, et al. Predicting fruit and vegetable consumption in long-haul heavy goods vehicle drivers: application of a multi-theory, Dual-phase model and the contribution of past behaviour. Appetite 2018;121:326-36.

31 Caudwell KM, Keech JJ, Hamilton K, et al. Reducing alcohol consumption during pre-drinking sessions: testing an integrated behaviour-change model. Psychol Health 2019;34:106-27.

32 Galli F, Chirico A, Mallia L, et al. Active lifestyles in older adults: an integrated predictive model of physical activity and exercise. Oncotarget 2018:9:25402-13.

33 Hamilton K, Fleig L, Henderson J, et al. Being active in pregnancy: Theory-based factors associated with physical activity among pregnant women. Women Health 2019;59:213-28.

34 Shannon S, Breslin G, Haughey T, et al. Predicting student-athlete and non-athletes' intentions to self-manage mental health: Testing an integrated behaviour change model. Ment Health Prev 2019;13:92-9.

35 Hamilton K, Gibbs I, Keech JJ, et al. Reasoned and implicit processes in heavy episodic drinking: an integrated dual-process model. Br J Health Psychol 2020;25:189-209.

36 Phipps DJ, Hagger MS, Hamilton K. Predicting limiting 'free sugar' consumption using an integrated model of health behavior. Appetite 2020;150:104668.

37 Kwasnicka D, Vandelanotte C, Rebar A, et al. Comparing motivational, self-regulatory and habitual processes in a computertailored physical activity intervention in hospital employees - protocol for the PATHS randomised controlled trial. BMC Public Health 2017;17:1-16.

38 Michie S, Richardson M, Johnston M, et al. The behavior change technique taxonomy (V1) of 93 hierarchically clustered techniques: building an international consensus for the reporting of behavior change interventions. Ann Behav Med 2013;46:81-95.
39 Prestwich A, Sniehotta FF, Whittington C, et al. Does theory influence the effectiveness of health behavior interventions? meta-analysis. Health Psychol 2014;33:465-74.

40 French SD, Green SE, O'Connor DA, et al. Developing theoryinformed behaviour change interventions to implement evidence into practice: a systematic approach using the theoretical domains framework. Implement Sci 2012;7:1-8.

41 Michie S, Abraham C, Whittington C, et al. Effective techniques in healthy eating and physical activity interventions: a meta-regression. Health Psychol 2009;28:690-701.

42 Dombrowski SU, Sniehotta FF, Johnston M, et al. Optimizing acceptability and feasibility of an evidence-based behavioral intervention for obese adults with obesity-related co-morbidities or additional risk factors for co-morbidities: an open-pilot intervention study in secondary care. Patient Educ Couns 2012;87:108-19.

43 Hagger MS, Chatzisarantis NLD, Biddle SJH. The influence of autonomous and controlling motives on physical activity intentions within the theory of planned behaviour. Br J Health Psychol 2002; 7:283-97.

44 Martins RK, McNeil DW. Review of motivational interviewing in promoting health behaviors. Clin Psychol Rev 2009;29:283-93.

45 Friederichs SAH, Bolman C, Oenema A, et al. Exploring the working mechanisms of a web-based physical activity intervention, based on self-determination theory and motivational interviewing. Internet Interv 2016;3:8-17.

46 Carraro N, Gaudreau P. Spontaneous and experimentally induced action planning and coping planning for physical activity: a metaanalysis. Psychol Sport Exerc 2013;14:228-48.

47 Sniehotta FF, Scholz U, Schwarzer R. Action plans and coping plans for physical exercise: a longitudinal intervention study in cardiac rehabilitation. Br J Health Psychol 2006;11:23-37.

48 Hendriks T, Schotanus-Dijkstra M, Hassankhan A, et al. The efficacy of multi-component positive psychology interventions: a systematic review and meta-analysis of randomized controlled trials. $J$ Happiness Stud 2020;21:357-90.

49 Labarthe DR, Kubzansky LD, Boehm JK, et al. Positive cardiovascular health: a timely convergence. J Am Coll Cardiol 2016;68:860-7.

50 Chiesa A, Serretti A. Mindfulness-based stress reduction for stress management in healthy people: a review and meta-analysis. J Altern Complement Med 2009;15:593-600.

51 Meyer JD, Torres ER, Grabow ML, et al. Benefits of 8-wk mindfulness-based stress reduction or aerobic training on seasonal declines in physical activity. Med Sci Sports Exerc 2018;50:1850-8.

52 Free C, Phillips G, Felix L, et al. The effectiveness of M-health technologies for improving health and health services: a systematic review protocol. BMC Res Notes 2010;3:1-7.

53 Lustria MLA, Cortese J, Noar SM, et al. Computer-tailored health interventions delivered over the web: review and analysis of key components. Patient Educ Couns 2009;74:156-73.

54 Neville L, O'Hara B, Milat A. Computer-tailored physical activity behavior change interventions targeting adults: a systematic review. Int J Behav Nutr Phys Act 2009;6:30-12.

55 Bennett GG, Glasgow RE. The delivery of public health interventions via the Internet: actualizing their potential. Annu Rev Public Health 2009;30:273-92.

56 Kroeze W, Werkman A, Brug J. A systematic review of randomized trials on the effectiveness of computer-tailored education on physical activity and dietary behaviors. Ann Behav Med 2006;31:205-23.

57 Kelders SM, Kok RN, Ossebaard HC, et al. Persuasive system design does matter: a systematic review of adherence to web-based interventions. J Med Internet Res 2012:14:e152.

58 Mohr DC, Cuijpers P, Lehman K. Supportive accountability: a model for providing human support to enhance adherence to eHealth interventions. J Med Internet Res 2011;13:e30.

59 Sattar N. Do pregnancy complications and CVD share common antecedents? Atheroscler Supp/ 2004:5:3-7.

60 Heida KY, Bots ML, de Groot CJ, et al. Cardiovascular risk management after reproductive and pregnancy-related disorders: a Dutch multidisciplinary evidence-based guideline. Eur J Prev Cardiol 2016;23:1863-79.

61 Mosca L, Benjamin EJ, Berra K, et al. Effectiveness-based guidelines for the prevention of cardiovascular disease in women--2011 update: a guideline from the american heart association. Circulation 2011:123:1243-62.

62 Bellamy L, Casas J-P, Hingorani AD, et al. Pre-Eclampsia and risk of cardiovascular disease and cancer in later life: systematic review and meta-analysis. BMJ 2007;335:974.

63 Ahmed R, Dunford J, Mehran R, et al. Pre-Eclampsia and future cardiovascular risk among women: a review. J Am Coll Cardiol 2014:63:1815-22. 
64 Magee LA, von Dadelszen P. Pre-Eclampsia and increased cardiovascular risk. BMJ 2007;335:945-6.

65 Sattar N, Greer IA. Pregnancy complications and maternal cardiovascular risk: opportunities for intervention and screening? BMJ 2002;325:157-60.

66 Newstead J, von Dadelszen P, Magee LA. Preeclampsia and future cardiovascular risk. Expert Rev Cardiovasc Ther 2007;5:283-94.

67 Chan A-W, Tetzlaff JM, Gøtzsche PC, et al. Spirit 2013 explanation and elaboration: guidance for protocols of clinical trials. BMJ 2013;346:e7586.

68 Benschop L, Duvekot JJ, Versmissen J, et al. Blood pressure profile 1 year after severe preeclampsia. Hypertension 2018;71:491-8.

69 Hardcastle SJ, Fortier M, Blake N, et al. Identifying content-based and relational techniques to change behaviour in motivational interviewing. Health Psychol Rev 2017:11:1-16.

70 Gharad B, Dean K, Scott N. Commitment devices. Annu Rev Econom 2010;2:671-98.

71 Grossman P, Niemann L, Schmidt S, et al. Mindfulness-based stress reduction and health benefits. A meta-analysis. J Psychosom Res 2004;57:35-43.
72 Imboden MT, Nelson MB, Kaminsky LA, et al. Comparison of four Fitbit and Jawbone activity monitors with a research-grade ActiGraph accelerometer for estimating physical activity and energy expenditure. Br J Sports Med 2018;52:844-50.

73 Feter N, Dos Santos TS, Caputo EL, et al. What is the role of smartphones on physical activity promotion? A systematic review and meta-analysis. Int J Public Health 2019;64:679-90.

74 Davies CA, Spence JC, Vandelanotte C, et al. Meta-Analysis of internet-delivered interventions to increase physical activity levels. Int J Behav Nutr Phys Act 2012;9:52.

75 van Buuren S, Boshuizen HC, Knook DL. Multiple imputation of missing blood pressure covariates in survival analysis. Stat Med 1999;18:681-94.

76 Jakobsen JC, Gluud C, Wetterslev J, et al. When and how should multiple imputation be used for handling missing data in randomised clinical trials - a practical guide with flowcharts. BMC Med Res Methodol 2017;17:1-10.

77 WMA. Declaration of Helsinki. Fortaleza, Brazil, 2013. 